\title{
Depression in Parkinson's disease: Health risks, etiology, and treatment options
}

\author{
Pasquale G Frisina ${ }^{1,2}$ \\ Joan C Borod ${ }^{3,4}$ \\ Nancy S Foldi ${ }^{3,5}$ \\ Harriet R Tenenbaum ${ }^{6}$ \\ 'Leir Parkinson's Disease Program, \\ The Jewish Home and Hospital \\ Lifecare System, New York, NY, \\ USA; ${ }^{2}$ Department of Geriatrics and \\ Adult Development, Mount Sinai \\ School of Medicine, New York, NY, \\ USA; ${ }^{3}$ Department of Psychology, \\ Queens College and The Graduate \\ Center of the City University of New \\ York (CUNY), Flushing, NY, USA; \\ ${ }^{4}$ Department of Neurology, Mount \\ Sinai School of Medicine, New York, \\ NY, USA; ${ }^{5}$ Department of Medicine, \\ Winthrop-University Hospital, State \\ University of New York, Stony Brook \\ School of Medicine; ${ }^{6}$ Department \\ of Psychology, Kingston University, \\ Kingston upon Thames, UK
}

Correspondence: Pasquale G Frisina The Jewish Home and Hospital Life Care System, I20 West 106th Street, New York, NY 10025, USA

Tel +l 2128705957

Email pfrisina@jhha.org

\begin{abstract}
Depression is found in about $30 \%-40 \%$ of all patients with Parkinson's disease (PD), but only a small percentage (about 20\%) receive treatment. As a consequence, many PD patients suffer with reduced health-related quality of life. To address quality of life in depressed PD patients, we reviewed the literature on the health correlates of depression in PD (eg, cognitive function), etiology of depression in PD, and treatment options (ie, antidepressants, electroconvulsive therapy, and psychotherapy). The current review is unique in its focus on psychosocial aspects, as well as neuropathological factors, of depression in PD. Overall, we conclude that neurochemical (eg, serotonin) and psychosocial factors (eg, coping style, self-esteem, and social support) contribute to the affective disturbances found in this neuropsychiatric population. Therefore, we recommend that a multidisciplinary (eg, pharmacotherapeutic, psychoeducational, and/or psychotherapeutic) approach to treatment be taken with depressed PD patients.
\end{abstract}

Keywords: depression, Parkinson's disease, health outcomes, treatment options

Parkinson's disease (PD) is a neurodegenerative disorder caused by the loss of dopaminergic neurons in the midbrain, which results in dysfunction of the nigrostriatal system producing alterations in movement such as tremor, bradykinesia, rigidity, and postural abnormalities. PD is generally characterized as a movement disorder, but depression occurs frequently in this population with a prevalence estimated at 30\%-40\% (Cummings 1992; Slaughter et al 2001; Zgaljardic et al 2003). Unfortunately, however, only $20 \%$ of all depressed PD patients receive treatment for their psychological status (Mayeux et al 1986; Huber et al 1988; Starkstein et al 1990). If depression is left untreated, there is an increased risk for greater disability and reduced quality of life (Edwards et al 2002).

One reason why depression is undertreated in PD is due to the patient's overshadowing concern for his/her physical relative to psychological health. Brod and colleagues (1998) have provided some evidence for this notion by observing that only $11 \%$ of depressed PD patients rated their psychological condition as a concerning health problem even though a majority of them were depressed. Similarly, depression failed to rank as one of the 20 most bothersome symptoms experienced by depressed PD patients (Scott et al 2000). Thus, even when PD patients are suffering from affective disturbances, they are more likely to complain about the burden of their motor disability. Therefore, physicians should regularly screen for depression when treating PD patients. However, it is often a difficult diagnosis as many of the cognitive (eg, decreased concentration), motor (eg, agitation and decreased facial expression), and somatic (eg, decreased energy and disturbed sleep) features of PD and depression overlap (Edwards et al 2002).

Treatment is also complicated by the lack of reliable and valid instruments for assessing depression in patients with PD (Edwards et al 2002). For instance, rating 
scales, such as the Beck Depression Inventory (BDI) (Beck et al 1961) and Hamilton Depression Rating Scale (HDRS) (Hamilton 1960), may over-diagnose depression in PD because the source of the somatic items could be influenced by parkinsonian symptoms (Leentjens et al 2000). However, the BDI and HDRS were tested for their diagnostic accuracy for depression in PD (Leentjens, Verhey, Lousberg et al 2000; Leentjens, Verhey, Luijckx et al 2000), and both scales showed adequate sensitivity (BDI $=67 \%$, and $\mathrm{HDRS}=83 \%$ ) and specificity (BDI $=88 \%$, and $\mathrm{HDRS}=95 \%$ ). Conversely, DSM-IV criteria are the most widely accepted method of evaluating depression in PD (Zesiewicsz et al 1999), but require a trained administrator and may take a significant amount of time to administer. Because it is easier to evaluate depression with the BDI than with DSM-IV criteria or the HDRS, the BDI should be considered as the preferred method for screening depression in PD. However, future research should assess the geriatric depression scale (Yesavage et al 1982) for its diagnostic accuracy in PD because it may provide better control for the somatic complaints that are associated with age and disease severity in PD.

Even when a diagnosis of depression is made accurately, it is not clear which treatment options (eg, psychotherapy or pharmacotherapy) are safe and effective for this population. Thus, in this article, we review the health risks, etiological factors, and treatment options for depression in PD towards improving health-related quality of life in PD patients suffering from depression. The current review is unique in its focus on psychosocial aspects, as well as neuropathological factors, of depression in PD.

\section{Depression and associated health risks in PD \\ Depression and cognition}

Cognitive deficits are common even in non-depressed and non-demented PD patients (for reviews, see Raskin et al 1990; Morrison et al 2000). However, certain aspects of cognition are more severely impaired in depressed PD patients (for reviews, see Emre 2003; Zgaljardic et al 2003). Unfortunately, these impairments can place depressed patients with PD at greater risk for developing dementia (Dalrymple-Alford et al 1994; Lombardi et al 2001). Dementia constitutes a negative health outcome in PD because it is associated with greater functional disability and institutionalization in nursing homes (Emre 2003). Moreover, mortality rates are higher for demented than non-demented PD patients (Mindham et al 1982). Thus, identification and treatment of PD patients with depression may prevent subsequent cognitive and functional decline.
Executive dysfunction is the type of cognitive impairment most closely related to the development of dementia in PD (Levy et al 2002). Indeed, depressed patients with PD have significantly greater impairments in attention and working memory (Uekermann et al 2003) and perform more poorly on neuropsychological measures of frontal/executive function (ie, Wisconsin Card Sorting Test and Raven Progressive Matrices) than non-depressed patients with PD (Kuzis et al 1997). Other studies have bolstered these findings in showing that depression at baseline increases the risk for developing dementia later in PD (Starkstein et al 1990; Stern et al 1993; Hughes et al 2000). Indeed, PD patients who were treated for depression evidenced less cognitive decline over a 3-4 year period relative to those who were not (Kremer and Starkstein 2000). The extent to which cognitive decline contributes to depression could help to understand functional disability and health-related quality of life in PD. Thus, treating depression in PD may help to prevent or delay the progression of cognitive and functional decline in this neuropsychiatric population.

\section{Depression and health-related quality of life}

The term health-related quality of life (HRQL) has been used to describe the distress and functional impairment produced by a chronic debilitating illness. ${ }^{1}$ Overall, research using generic and disease-specific scales has found motor disability to be an obvious and important determinant of reduced HQRL in PD (Peto et al 1995; Dodel et al 2001; Keranen et al 2003). However, there is also a growing literature that shows that even beyond motor disability, depression can be a risk factor for reduced HRQL in PD (Karlsen et al 1995; Hobson et al 1999; Schrag et al 2001).

Karlsen et al (1999) used the Nottingham Health Profile (NHP) to determine which clinical features reduce HRQL in PD. A generic quality of life questionnaire, the NHP, measures six dimensions - emotional reactions, energy, pain, physical mobility, sleep, and social isolation (Jenkinson et al 1988). Overall, depression was related to negative emotional reactions, low energy, pain, and social isolation but not to physical mobility problems and sleep disorders (Karlsen et al 1999). Nonetheless, other studies using PD-specific measures of HRQL have found a relationship between depression and these health outcomes (Hobson et al 1999; Schrag et al 2001).

One study that used a PD specific scale on HRQL (ie, PDQ-39) observed that depression was significantly associated with poorer quality of life in this population (Schrag 
et al 2001). Briefly, the PDQ-39 is a PD-specific, HRQL questionnaire that consists of eight health dimensions - mobility, activities of daily living, emotional well-being, stigma, social support, cognition, communication, and bodily discomfort (Peto et al 1995). Overall, depressed PD patients scored significantly lower than non-depressed PD patients on all eight dimensions of the PDQ-39, particularly on items that tapped emotional well-being and cognition. Furthermore, after controlling for motor disability severity, depression significantly predicted all areas of HRQL on the PDQ-39.

Additional evidence deriving from another diseasespecific scale has offered greater reliability to the notion that depression is a serious risk factor for reduced HRQL found in PD. De Boer and colleagues (1996) developed the PD Quality of Life Questionnaire (PDQL) to determine which clinical and demographic variables are related to HRQL in PD. It assesses four dimensions of health - parkinsonism, systemic symptoms, social functioning, and emotional functioning. Again, depression was the best predictor of poor HRQL along all four dimensions of the PDQL relative to age, disease severity, and cognitive impairment (Hobson et al 1999). Further, depression produced the poorest scores on the parkinsonian and emotional functioning subscales of the PDQL (Hobson et al 1999).

The studies (reviewed above) using the PDQ-39 and PDQL produced convergent results that depression predicted reduced HRQL (Hobson et al 1999; Schrag et al 2001). In a recent paper on the psychometric properties of the PDQ-39 and PDQL (Marinus et al 2002), the PDQ-39 was deemed the most appropriate instrument for measuring HRQL "because it has been tested most thoroughly, has adequate clinimetric characteristics, has been used in the largest number of studies, and is available in many languages" ( $p$ 248). Thus, the PDQ-39 can be adopted as an instrument for studying depression and HRQL in PD.

Depression should be considered a risk factor for poor HRQL in PD, thus, it is conjectured that HRQL, cognitive function, and emotional-well-being would potentially improve once depression is alleviated in PD. However, what is not clear are the factors that contribute to depression in PD and which treatment options are safe and effective for use in this neuropsychiatric population.

\section{Etiology of depression in PD}

There have been several explanations for why depression occurs in PD. A psychological explanation suggests that depression is a reaction to the stress of coping with a chronic and progressively disabling disease. A biomedical perspective argues that depression is a primary neurochemical consequence of the neurodegenerative process of PD, attributing depression in PD to deficits in dopaminergic, noradrenergic, and/or serotonergic systems (Hornykiewicz 1982; Cummings 1993). Evidence from studies that have examined disease correlates (eg, motor severity, duration, and functional disability) of $\mathrm{PD}$, prevalence rates, and brain abnormalities between depressed and non-depressed PD patients have supported both models and are described below.

\section{Correlates of depression within PD}

According to the psychological-reactivity model, it is hypothesized that depression will be positively correlated with severity, duration, and disability factors in PD. Studies examining these correlates of depression in PD have not provided consistent evidence for the psychological-reactivity model. For instance, studies that have examined PD disease duration and depression did not find a significant relation between the two variables (Warburton 1967; Celesia and Wannamaker 1972; Lesser et al 1979; Mayeux et al 1981,1984; Dakof and Mendelsohn 1989; MacCarthy and Brown 1989; Schrag et al 2001). Whereas some studies found a significant relationship between PD severity and depression (Hoehn et al 1976; Mindham et al 1976; Singer 1976; Gotham et al 1986; Dakoff and Mendelsohn 1989; Fleminger 1991; Schrag et al 2001), others have not (Warburton 1967; Celesia and Wannamaker 1972; Mayeux et al 1981; Mayeux 1984; Huber et al 1988; MacCarthy Brown 1989).

Findings relating depression to functional disability are also inconsistent. For instance, several studies have found that depression is significantly and positively correlated with disability in PD (Gotham et al 1986; Brown et al 1988; Ehman et al 1990; Menza and Mark 1994; Tandberg et al 1997; Schrag et al 2001). However, depression does not improve in $\mathrm{PD}$ patients once disability is reduced following levodopa therapy (Goodwin et al 1970; Cheifetz et al 1971; Marsh and Markhan 1973; Hoehn et al 1976; Lesser et al 1979; Mayeux et al 1981). Therefore, conflicting results do not provide a clear indication that depression is simply a reaction to the disabling aspects of PD.

\section{Biomedical perspective and brain imaging}

One aspect of the biomedical model argues that depression may be a sequela of changed brain chemistry that is separate from the dopaminergic deficiency associated with PD motor symptoms (Marsh and Markham 1973; Mayeux 1984; Mayeux 
et al 1984). This premise is based on the observation that levels of cerebrospinal fluid 5-HIAA, a metabolite of serotonin (5-HT), are reduced in depressed PD patients (Mayeux et al 1984, 1986). Initially, critics of this hypothesis reported that even non-depressed PD patients showed metabolite reductions (Kostic et al 1987), suggesting that either CSF metabolites were inaccurate measures of changes in brain 5-HT or that depressed PD patients were not qualitatively different from non-depressed PD patients. More recent neuroimagining and neuropharmacological research has been used to better understand the biological etiology of PD depression.

Neuroanatomical differences between depressed and non-depressed PD patients have been studied (Mayberg et al 1990; Mayberg and Solomon 1995; Sagar 1999). Depressed PD patients show metabolic abnormalities during PET scans in both the caudate and inferior frontal cortices as compared to non-depressed PD patients and aged-matched healthy controls (Mayberg et al 1990). PET studies have also shown lower metabolic activity in the prefrontal cortex in depressed relative to non-depressed PD patients (Paulus and Trenkwalder 1998). Though these studies point to a biological etiology of PD depression, one criticism is that while depressed and non-depressed PD patients are compared, these studies do not directly compare depressed PD patients to non-neurologically ill depressed patients. This leaves open the possibility that depression alone could also contribute to metabolic deficits, rather than the effect of PD.

Evidence from non-neurologically depressed patients has shown metabolic abnormalities in the same area of the brain (ie, prefrontal cortex) that is also affected in depressed patients with PD (Rubin et al 1994; Brody et al 2001; Martin et al 2001). Moreover, psychotherapy is useful in reducing depression and in increasing metabolic activity (assessed through PET) in the frontal cortex, temporal lobe, basal ganglia, and cingulate gyrus in non-neurological depressed patients (Martin et al 2001). Thus, there is a potential contribution of psychosocial factors to neuroanatomical differences observed between depressed and non-depressed PD patients (Mayberg et al 1990; Mayberg and Solomon 1995; Sagar 1999). The differentiation of abnormalities among depressed PD patients, non-depressed PD patients, and nonneurologically ill patients with depression has to be made, and understood in the context of structural neuroanatomy, metabolic activity, and therapeutic treatment response.

\section{Depression in PD versus other illnesses}

Whether depression is found more frequently in PD relative to other non-neurological illnesses is unclear. If PD patients are at greater risk for developing depression, then the affective disturbances found in PD can be related to neurological disease as opposed to a psychosocial stress response in reaction to having a chronic and disabling illness. Several studies examined rates of depression between PD patients and other disabled clinical populations. Again, the collective evidence does not support either a biomedical or psychological interpretation. For instance, three separate studies found that PD patients were significantly more depressed than paraplegics, amputees, and patients suffering from various medical/surgical conditions (Wartburton 1967; Horn 1974; Robins 1976). However, the comparison groups in these studies were not equivalent to the PD sample with respect to age and duration of illness.

Ehman et al (1990) compared levels of depression between patients with PD and rheumatoid arthritis (RA), and observed that depression was higher for the PD group. This finding could suggest that depression in PD may be caused by endogenous factors rather than a psychological reaction to coping with a physically debilitating disorder. However, the two groups were different with respect to disease duration, with the PD group duration lasting 3.5 years whereas the RA group was ill for over 14 years. Given that other studies have shown that psychological disturbances tend to occur more frequently in the early stages of chronic illness because the patient has not yet learned how to cope effectively with their disease (Meyerowitz 1980), the greater rates of depression found in PD relative to RA patients (Ehman et al 1990) may be attributed to the PD patients not having enough time to develop a more adaptive means of coping with their illness. Singer (1976) suggested that the longer PD patients have the disease, the more stoically they accept their symptoms. Thus, an important variable that may distinguish depressed from non-depressed PD patients is how the individual copes with their chronic illness over time (Brown et al 1988).

\section{Psychosocial reactivity and depression}

Psychosocial variables, such as coping style and amount of social support, may predict depression more reliably than severity, illness duration, and functional disability (Brown and McCarthy 1989; Dakoff and Mendelson 1989; Ehman et al 1990; Frazier 2000). For instance, Brown and McCarthy (1989) examined the influence of psychosocial variables in predicting depression in PD patients. Overall, the best predictors of depression were functional disability, low self-esteem, and avoidant coping. These variables accounted for $46 \%$ of the variance in depression. Furthermore, positive affect and cognitive coping (eg, acceptance of illness) significantly predicted 
lower depression. This study found that disease-related variables (eg, severity and functional disability) do not predict a large portion of the variance in the depression that is experienced in PD. The observed inconsistent findings suggest that the reactivity model is in need of revision and should also include psychosocial variables (Dakoff and Mendelson 1989; Ehman et al 1990; Frazier 2000).

Evidence supports the notion that psychosocial factors reliably predict depression in PD (Ehman et al 1990). After controlling for functional disability, PD patients were significantly more depressed than RA patients. Again, this finding could be interpreted as evidence for the biological model of depression in PD. However, coping style was also examined; the RA group used significantly more cognitive coping strategies in dealing with their disease than the PD group. Instead, the PD group used more avoidant coping to manage their disease. Interestingly, when PD patients utilized cognitive coping strategies, there were significant reductions in depression, improvements in disability, and more frequent engagements in socialization. Thus, this study found that disease-related variables (eg, functional disability) are not sufficient for a clear picture of the etiology of PD depression and that psychosocial variables, such as coping style, are important in understanding the reactive nature of depression in PD.

One important question that can be raised from the above psychosocial literature is whether PD patients, who may perseverate or lack the ability to be flexible, have difficulty adopting more malleable coping styles. Relevant to this question, research shows that PD patients have a tendency to maintain stable coping patterns across the trajectory of their illness even when such strategies lead to poor mental and physical health outcomes (Frazier 2002). For instance, the majority of PD patients continued to use maladaptive coping strategies (eg, active coping for physical stressors) rather than shift to adaptive coping strategies (eg, emotion-regulation coping) in the face of progressing motor and functional disability (Frazier 2002). Although it was less common, PD patients who displayed flexibility in coping strategies over time evidenced better health outcomes. Thus, PD patients, on the whole, may benefit from a psychosocial intervention that educates them on utilizing appropriate coping strategies for various stressors associated with the progressive and unpredictable nature of PD. Additionally, because the majority of PD patients utilized stable and inflexible (ie, dispositional) coping strategies, research directed toward differentiating PD from other movement disorders and other non-neurologically ill populations (eg, rheumatoid arthritis) could be conducted.
These comparisons would directly test whether PD patients by nature of their neurological disease have a particular coping style that contributes to their depression.

In summary, as evidence does not permit discrete choice between the biomedical or psychological position with respect to the etiology of $\mathrm{PD}$ depression, a more parsimonious perspective should include a combination of endogenous (neurochemically based) and exogenous (environmental) factors (eg, Poewe and Seppi 2001). Therefore, we further examined the literature on treatment options for PD depression to understand its etiology towards improving quality of life for this neuropsychiatric population.

\section{Treatment options for depression in PD}

\section{Tricyclic antidepressants}

Depression in PD has been treated effectively with tricyclic antidepressants (TCAs). Early work prior to L-dopa use may not be comparable to research after its introduction in 1975. Strang (1965) conducted a double-blind study on the effects of imipramine (150-250 mg/day) in PD and found that depression improved for $60 \%$ of the patients. Moreover, this study found that antidepressant therapy had a beneficial effect on rigidity, tremors, and akinesia. Supportive studies showed that motor deficits and depression were improved using a double-blind study on the effects of desipramine (100 mg/day) in PD: reductions in rigidity and tremors were evident in patients whose depression responded favorably to antidepressants (Laitnen 1969). These results suggest that TCAs can be used effectively for treating both depression and motor disability in PD. The association between depression and motor improvement is that imipramine (Strang 1965) and desipramine (Laitnen 1969), as well as nortriptyline (Anderson et al 1980) and amitriptyline (Indaco and Carrieri 1988), block the reuptake of norepinephrine (Maxmen and Ward 1995). The mechanism for TCAs on norepinephrine (NE) is important because of the link of NE to parkinsonian symptoms and to dopamine (DA) levels within the basal ganglia, and the progressive loss of noradrenergic neurons in the locus coeruleus of PD patients (Gesi et al 2000). Animal models of PD show that drugs that increase the concentration of NE in the locus coeruleus (eg, 2-methoxy idazoxan) also reduce parkinsonian symptoms and increase DA levels in the basal ganglia of rats (Srinivasan and Schmidt 2003, 2004). In terms of depression, TCAs also block 5-HT reuptake in the raphe system and frontal cortex (Bel and Artigas 1996). Therefore, TCAs can improve motor and affective function in PD by blocking NE reuptake in two distinct pathways (ie, nigrostriatal [DA] and raphe [5-HT] systems). 
Whereas TCAs may be useful in treating depression and motor functioning (Strang 1965; Laitinen 1969; Andersen et al 1980), many patients cannot tolerate them although some side effects of the TCAs in lower doses are used to treat excess salivation and sleep derangement in PD patients. Adverse effects of TCAs include cardiac arrhythmias, orthostatic hypotension, fatigue, and memory impairment. At high doses, TCAs can produce confusional states, seizures, and delirium in depressed PD patients. Because selective serotonin reuptake inhibitors (SSRIs) have a lower side effect profile in patients without PD, physicians have more recently been prescribing SSRIs over TCAs for depressed PD patients (Richard and Kurlan 1997). However, it is important to note that the safety and efficacy of SSRIs have not been established for depressed PD patients.

\section{Serrotonin re-uptake inhibitors}

There have been several clinical trials examining the effects of SSRIs in PD. Hauser and Zesiewicz (1997) conducted a 7-week open-label trial that examined the effects of sertraline $(25-50 \mathrm{mg} / \mathrm{d})$ in 15 depressed PD patients. Overall, there was a significant decrease in depression levels between baseline and post-testing. Unlike the TCA studies described above, sertraline did not improve motor performance measured by the Unified Parkinson's Disease Rating Scale (UPDRS) (Fahn et al 1987). There have also been two open-labeled clinical trials examining the effects of paroxetine for depression in PD. Once again, these studies found that SSRIs were able to reduce depression, but there was also evidence of a deterioration of motor symptoms in many of the PD patients (Ceravolo et al 2000; Tesei et al 2000). Thus, SSRIs can be effective in treating depression in PD but without the salutary benefits that were seen with TCAs on motor functioning (Strang 1965; Laitinen 1969; Anderson et al 1980; Goetz et al 1984; Klaasen et al 1995) or even an increase in motor symptoms. The question remains as to whether or not SSRIs can exacerbate parkinsonian symptoms.

Research regarding the use of SSRIs in PD shows deterioration in motor symptoms in some studies (Steur 1993; Jimenez et al 1994; Ceravolo et al 2000), while not in others (Caley and Friedman 1992; Montastruc et al 1995; Hauser and Zesiewicz 1997). These studies used low constraint research methods (ie, case reports and open-label trials). More recent placebo-controlled trials have assessed the effects of SSRIs on depressive and motor symptoms in PD (Wermuth et al 1998; Rampello et al 2002; Leenjens et al 2003). Wermuth et al (1998) conducted a placebo-controlled trial on PD patients suffering from major depression. The SSRI citalopram or placebo was administered to an average of 10 subjects in each group over the course of 52 weeks. There were no significant group differences on measures of depression (ie, HDRS), motor function (ie, UPDRS), or adverse side effects (UKU Side Effects Scale; Lingjaerde et al 1987). Of note, at study outset, $60 \%$ of the sample consisted of patients with severe recurrent brief depression, which lasted for 2-3 days. Perhaps a treatment effect was not observed because depression would have subsided in the majority of patients after several days. Thus, this randomized placebo-controlled trial can be criticized for containing sampling error. Leentjens et al (2003) also conducted a study using the SSRI citalopram $(n=6)$ or placebo $(n=4)$ on severely depressed PD patients. Once again, there were no significant group differences in measures of depression (ie, Montgomery-Asberg Depression Rating Scale (MADRS)) (Montgomery and Asberg 1979) or motor function (ie, UPDRS). Because this study contained only 12 severely depressed PD patients, the non-significant findings could have been due to lack of statistical power.

Rampello et al (2002) observed that citalopram improved motor performance (ie, bradykinesia and finger tapping speed) for both depressed $(n=18)$ and non-depressed ( $n=14)$ PD patients relative to a non-depressed placebo $(n=14)$ control group. Depression was significantly reduced for the depressed PD subgroup. The researchers argued that improvements in depression and motor function occurred because of the ability of SSRIs to enhance mesolimbic serotonin levels and to simultaneously reinforce dopaminergic release in cell bodies of the nigrostriatal system via 5-HT2a and 5-HT4 postsynaptic and/or 5-HT3 presynaptic receptors. These findings support the biological basis of depression in PD (Hornykiewicz 1982; Cummings 1993) and that SSRIs, such as citalopram, might provide salutary health benefits to depressed and non-depressed PD patients.

\section{Monoamine-oxidase inhibitors}

The biomedical approach has attributed PD-related depression to a deficit in dopaminergic (Mayeux 1992), noradrenergic (Hornykiewicz 1982), and serotonergic brainstem ascending systems (Hornykiewicz 1982; Mayeux et al 1984). As a result, inhibition of monoamine oxidase (MAO) does not seem like an unreasonable approach to the treatment of PD depression. The two forms of MAO inhibitors were relevant, MAO-A (selective for noradrenaline and serotonin metabolism for treatment of depression) and MAO-B (selective for DA and used in PD treatment), especially early on to postpone progression of disease. If used in high doses, MAO-B inhibitors will be active for A and B pathways. 
When selegiline is dosed at $10 \mathrm{mg}$ a day or less in PD, it can serve as an irreversible inhibitor of brain MAO-B enzyme. Accordingly, selegiline at $10 \mathrm{mg}$ a day may increase DA levels, which in turn can improve motor function in PD patients (Parkinson Study Group 1993). Thus, selegiline, at its usual clinical dose, should not exert an antidepressant effect because it selectively inhibits MAO-B (and not MAO-A) enzymes in the adult human brain. However, selegiline was initially introduced as an antidepressant because the drug can also act as an MAO-A inhibitor when given at slightly higher doses (Sunderland et al 1985).

Quitkin and colleagues (1984) tested the effects of selegiline (20 mg/day) on 17 atypical depressives without PD and found that improvement occurred in $60 \%$ of the patients. Mann et al (1989) conducted a double-blind placebocontrolled trial testing the effects of selegiline on patients suffering from primary depression. Depression was significantly reduced in patients assigned to the selegiline group relative to those assigned to the placebo control group. Additionally, significantly greater reduction of depression scores occurred with higher $(>30 \mathrm{mg})$ than with lower $(<30 \mathrm{mg})$ doses of selegiline. A more recent placebo-controlled trial revealed that transdermal selegiline (20 mg/day) administered for 6 weeks was an effective and well-tolerated treatment for 177 adult outpatients suffering from major depression (Bodkin and Amsterdam, 2002). Therefore, selegiline could serve as an efficacious antidepressant at higher doses in depressed patients with PD. However, its use at higher doses in PD is sparse, and the focus of selegiline research has been restricted to MAO-B inhibition.

\section{Dopamine replacement}

Some literature implicates DA agonist's utility in the treatment of depression. For instance, D2/D3 agonists (eg, pramipexole and pergolide) have reduced depression in non-neurologically ill patients and improved motor function in PD patients (Corrigan et al 2000; Reichmann et al 2003; Rektorova et al 2003). To date, there have been no known studies examining the effects of dopaminergic antidepressants (eg, buproprion or velaxefine) in PD. Because DA agonists have been shown to reduce depression and improve motor function in PD, more research is needed to examine whether selegiline and dopaminergic antidepressants can serve as both antidepressant and antiparkinsonian agents in PD.

A meta-analysis conducted in Parkinson's disease (Weintraub et al 2005) examined the effects of antidepressants on PD-related depression and obtained a large effect size for depression outcomes in both the antidepressant $(d=1.34)$ and placebo control group $(d=1.19)$. Further, the researchers did not observe a significant between-group difference, $Q b(1)=0.43, p=0.51$. They concluded that antidepressant treatment has a "non-specific" effect on depression in PD, which may be confounded because the effect size data from their control group was derived only from two SSRI studies that possibly contained sampling error (Wermuth et al 1998; Leenjens et al 2003). Moreover, the researchers excluded the TCA antidepressant class from their analysis which may have further limited their ability to detect a significant treatment effect between the antidepressant and placebo groups. As TCAs can be effective in treating depression and improving motor function in PD (Strang 1965; Laitnen 1969; Anderson et al 1980; Indaco and Carrieri 1988), physicians in clinical care might consider using TCAs for certain situations in depressed PD patients.

\section{Electroconvulsive therapy}

The potential benefits of electroconvulsive therapy (ECT) have been explored as a treatment modality for PD patients suffering from depression. A literature review conducted between 1975 and 1991 (Faber and Trimble 1991) provided evidence that depression can be reduced in most PD patients treated with ECT. Approximately $70 \%$ of PD patients showed improvement in psychiatric disturbances (including depression) across the 21 studies reviewed. In another study conducted on 25 depressed PD patients, depression was significantly reduced following ECT (Moellentine et al 1998). ECT can serve as a viable treatment option for depressed PD patients as the electrically induced seizures can increase both norepinephrine and serotonin levels in the brain (Poewe and Seppi 2001). Because norepinephrine increases DA levels in the basal ganglia and reduces motor symptoms (Gesi et al 2000; Srinivasan and Schmidt 2003, 2004), both affective and motor functions may be improved in PD via ECT. In support of this notion, several studies have found that ECT improved motor symptoms (Douyone et al 1989; Friedman and Gordon 1992; Mollentine et al 1998; Strome et al 2007) and depressed mood in PD patients (Faber and Trimble 1991).

Although these positive outcomes seem to suggest that ECT can serve as an alternative treatment to antidepressant therapy, risks for developing side effects such as delirium (Moellentine et al 1998) can occur with ECT use in PD. ECT may not be a first line therapy unless PD patients are self-destructive and/or non-responsive to drug therapy (Kim and Hershey 1988). The majority of depressed PD patients tend to suffer from mild to moderate depression and are 
not suicidal (Slaughter et al 2001), so that safe and effective treatments still rely on pharmacological or behavioral treatments. Consequently, ECT is not appropriate for most PD patients.

\section{Psychotherapy}

Consensus among physicians and researchers posits that psychotherapy can be a helpful treatment modality for PD patients, in general, and especially for those individuals who are suffering from mild or moderate depression (Dakof and Mendelsohn 1986; Poewe and Luginger 1999; Kremmer and Starkstein 2000; Slaughter et al 2001). For instance, behavioral therapy around the time of PD diagnosis and during advancing stages of the illness can adequately control for mood disorders in many PD patients (Poewe and Luginger 1999). Brown and Jahanshashi (1995) suggested that a psychotherapy program should be adapted to each PD patient to prevent and treat the depression that is associated with the disease. These researchers further suggested that the program should focus on disability and handicaps produced by PD and should be continued throughout the course of the illness (Brown and Jahanshashi 1995).

Whereas the importance of psychotherapy appears to be well recognized by the medical community, surprisingly, a literature search of the Medline and Psych-Info abstract indices generated only two experimental studies on the effects of a behavioral-cognitive intervention of any kind for PD patients suffering from affective disturbances (Ellgring et al 1993; Trend et al 2002). Ellgring et al (1993) systematically explored whether PD patients would respond favorably to a psychological intervention. Overall, counseling aimed at facilitating cognitive restructuring was able to reduce overall stress, improve social functioning, and increase acceptance of the illness. Whether this intervention would have improved depression, in particular, in this sample of PD patients is unclear because it was not assessed as a health outcome by the researchers (Ellgrig et al 1993).

Trend et al (2002) observed that depression scores were significantly reduced for PD patients after receiving relaxation training and individual talks from a multidisciplinary team of therapists, including a PD nurse specialist, physiotherapist, occupational therapist, speech therapist, and care manager, over a 6-week period. Although improvements in mood at post-testing were noted, none of the patients could be classified as depressed because of their low baseline scores on a non-validated measure of depression (ie, the Hospital Anxiety and Depression Scale) in PD. Therefore, the efficacy of behavioral-cognitive therapy has yet to be established for depressed PD patients.

\section{Conclusions}

Delayed treatment of depression in PD will lead to reduced quality of life. We believe that a multidisciplinary (eg, pharmacotherapy, psychoeducational, and/or psychotherapy) approach to treatment should be assumed. Nonetheless, before PD patients can be treated, they need to be accurately diagnosed with depression, and to reiterate, it is often difficult to diagnose depression in this clinical population because many of the symptoms of PD and depression overlap (Edwards et al 2002). Programs of research that focus on the measurement of depression in PD are urgently needed so that more patients can be actively identified and treated for their affective disturbances.

\section{Acknowledgments}

A portion of this work was conducted while Dr. Frisina was an Assistant Professor of Psychology at Iona College. This work was supported, in part, by a grant award from The LeirRidgefield Foundation to The Jewish Home and Hospital and by NIH R01 DC01150 subcontract and Professional Staff Congress-CUNY Research Award 64299-0033 to Queens College.

We would like to thank Barbara S. Koppel, M.D., for her insightful comments on the manuscript.

\section{Note}

${ }^{1}$ Currently, there are several scales that are used to measure health-related quality of life in PD, both generic (non-specific to PD) and specific to PD (Bergner et al 1981; Jenkinson et al 1988; Peto et al 1995).

\section{References}

Anderson J, Aabro E, Gulmann N, et al 1980. Anti-depressive treatment in Parkinson's disease: a controlled trial of the effect of nortriptyline in patients with Parkinson's disease treated with L-dopa. Acta Neurol Scand, 62:210-19.

Beck AT, Ward CH, Mendelson M, et al 1961. An inventory for measuring depression. Arch Gen Psychiatry, 4:561-71.

Bel N, Artigas F. 1996. In vivo effects of the simultaneous blockade of serotonin and norepinephrine transporters on serotonergic function. Microdialysis studies. J Pharmacol Exp Ther, 278:1064-72.

Bergner M, Bobbitt RA, Carter WB, et al 1981. The sickness impact profile: development and final revision of a health status measure. Med Care, 19:787-805.

Bodkin JA, Amsterdam JD. 2002. Transdermal selegiline in major depression: A double-blind, placebo-controlled, parallel-group study in outpatients. Am J Psychiatry, 159:1869-75.

Brod M, Mendoelsohn GA, Roberts B. 1998. Patients' experience of Parkinson's disease. J Gerontol, 53B:213-22.

Brody AL, Barsom MW, Bota RG, et al 2001. Prefrontal-subcortical and limbic circuit mediation of major depressive disorder. Semin Clin Neuropsychiatry, 6:102-12.

Brown R, MacCarthy B, Gotham AM, et al 1988. Depression and disability in Parkinson's disease: a follow-up study of 132 cases. Psychol Med, 18:49-55. 
Brown R, MacCarthy B. 1989. Psychosocial factors in Parkinson's disease. Br J Clin Psychol, 28:41-52.

Brown R, Jahanshashi M. 1995. Depression Parkinson's disease: a psychosocial viewpoint. Adv Neurol, 65:61-84.

Caley CF, Friedman JH. 1992. Does fluoxetine exacerbate Parkinson's disease? J Clin Psychiatry 53:278-82.

Celesia GG, Wanamaker WM. 1972. Psychiatric disturbances in Parkinson's disease. Dis Nerv System, 33:577-83.

Ceravolo R, Nuti A, Piccinni A et al 2000. Paroxetine in Parkinson's disease: effects on motor and depressive symptoms. Neurology, 55:1216-18.

Cheifetz DL, Garron DC, Leavitt F, et al 1971. Emotional disturbance accompanying the treatment of parkinsonism with L-DOPA. Clinic Pharmacol Therapeutics, 12:56-61.

Corrigan MH, Denahan AQ, Wright CE et al 2000: Comparison of pramipexole, fluoxetine, and placebo in patients major depression. Depress Anxi, 11:58-65.

Cummings JL. 1992. Depression and Parkinson's disease: a review. Am J Psychiatry, 149:443-54.

Cummings JL. 1993. Frontal-subcortical circuits and human behavior. Arch Neurol, 50:873-80.

Dakoff GA, Mendelsohn GA. 1986. Parkinson's disease: the psychological aspects of a chronic illness. Psychol Bull, 99:375-87.

Dakof GA, Mendelsohn GA. 1989. Patterns of adaptation to Parkinson's disease. Health Psychol, 8:355-72.

Dalrymple-Alford JC, Kalders AS, Jones RD, et al 1994. A central executive deficit in patients with Parkinson's disease. J Neurol Neurosurg Psychiatry, 57:360-7.

De Boer AG, Wijker W, Speelman JD, et al 1996. Quality of life in patients with Parkinson's disease: development of a questionnaire. J Neurol Neurosurg Psychiatry, 61:70-4.

Dodel RC, Berger K, Oertel WH. 2001. Health-related quality of life and healthcare necessity in patients with Parkinson's disease. Pharmacoeconomics, 19:1013-38.

Douyon R, Serby M, Klutchko B, et al 1989. ECT and Parkinson's disease revisted: a "naturalistic" study. Am J Psychiatry, 146:1451-55.

Edwards E, Kitt C, Oliver E, et al 2002. Depression and Parkinson's disease: a new look at an old problem. Depress Anxi, 16:39-48.

Ehmann TS, Beninger RJ, Gawel MJ, et al 1990. Depressive symptoms in Parkinson's disease: a comparison with disabled control subjects. $J$ Geriatr Psychiatry Neurol, 3:3-9.

Ellgring H, Seiler S, Perleth B, et al 1993. Psychosocial aspects of Parkinson's disease. Neurology, 43:S41-4.

Emre M. 2003. What causes mental dysfunction in Parkinson's disease? Mov Disord, 18:S63-71.

Faber R, Trimble M. 1991. Electroconvulsive therapy, Parkinson's disease and other movement disorders. Mov Disord, 6:293-03.

Fahn S, Elton RL. 1987. Members of the UPDRS development committee. United parkinson's disease rating scale. In Fahn S, Marsden, Calne DB, Goldstein M (ed). Recent developments in Parkinson's disease, 2nd ed. Florham Park (NJ): Macmillan Health Care Information. $p$ 153-64.

Fleminger S. 1991. Left-sided Parkinson's disease is associated with greater anxiety and depression. Psychol Med, 21:629-38.

Frazier LD. 2000. Coping with disease-related stressors in Parkinson's disease. The Gerontologist, 40:53-63.

Frazier LD. 2002. Stability and change in patterns of coping with Parkinson's disease. Int J Aging Hum Dev, 55:207-31.

Friedman J, Gordon N. 1992. Electroconvulsive therapy in Parkinson's disease: a report on five cases. Convulsive Therapy, 8:204-10.

Gesi M, Soldani P, Giorgi FS, et al 2000. The role of the locus coeruleus in the development of Parkinson's disease. Neurosci Biobehav Rev, 24:655-68.

Goetz CG, Tanner CM, Klawans HL. 1984. Bupropion in Parkinson's disease. Neurology, 34:1092-4.

Goodwin FK, Murphey DL, Brodie HK, et al 1970. L-DOPA, catecholamines, and behavior: a clinical and biochemical study in depressed patients. Biol Psychiatry, 2:341-66.
Gotham AM, Brown RG, Marsden CD. 1986. Depression in Parkinson's disease: a quantitative and qualitative analysis. $J$ Neurol Neurosurg Psychiatry, 49:381-9.

Hamilton M. 1960. A rating scale for depression. Journal of Neurology, Neurosurgery and Psychiatry, 23:56-62.

Hauser RA, Zesiewicz TA. 1997. Sertraline for the treatment of depression in Parkinson's disease. Mov Disord, 12:756-9.

Hobson P, Holden A, Meara J. 1999. Measuring the impact of Parkinson's disease with the Parkinson's disease quality of life questionnaire. Age Aging, 28:341-6.

Hoehn MM, Crowley TJ, Rutledge CO. 1976. Dopamine correlated of neurological and psychological status in untreated parkinsonism. J Neurol Neurosurg Psychiatry, 39:941-51.

Horn S. 1974. Some psychological factors in parkinsonism. J Neurol Neurosurg Psychiatry, 37:27-31.

Hornykiewicz O. 1982. Imbalance of brain monoamines and clinical disorders. Prog Brain Res, 55:419-21.

Huber SJ, Paulson GW, Shuttleworth EC. 1988. Depression in Parkinson's disease. Neuropsychiatry Neuropsychol Behav Neurol, 1:47-51.

Hughes TA, Ross HF, Musa S, et al 2000. A 10-year study of the incidence and factors predicting dementia in Parkinson's disease. Neurology, 54:1596-02.

Indaco A, Carrieri PD. 1988. Amitriptyline in the treatment of headache in patients with Parkinson's disease. Neurology, 38:1720-2.

Jenkinson C, Fitzpatrick R, Argyle M. 1988. The Nottingham health profile: an analysis of its sensitivity in differentiating illness groups. Soc Sci Med, 27:1411-14.

Jimenez-Jimenez FJ, Tejeiro J, Martinez-Junquera G, et al 1994. Parkinsonism exacerbated by paroxetine. Neurology, 44:240-6.

Karlsen KH, Larsen JP, Tandberg E, et al 1999. Influence of clinical and demographic variables on quality of life in patients with Parkinson's disease. J Neurol Neurosurg Psychiatry, 66:431-5.

Keranen T, Kaakkola S, Sotaniemi K, et al 2003. Economic burden and quality of life impairment increase with severity of PD. Parkinsonism Relat Disord, 9:163-8.

Kim KY, Hershey LA. 1988. Diagnosis and treatment of depression in the elderly. Int J Psychiatry Med, 18:211-21.

Klaassen T, Verhey FRJ, Sneijders GHJ, et al 1995. Treatment of depression in Parkinson's disease: a meta-analysis. J Neuropsychiatry Clin Neurosci, 7:281-6.

Kostic VS, Djuricic BM, Covickovic-Sternic N, et al 1987. Depression and Parkinson's disease: possible role of serotonergic mechanisms. J Neurol, 234:94-6.

Kremer J, Starkstein SE. 2000. Affective disorders In Parkinson's disease. Int Rev Psychiatry, 12:290-7.

Kuzis G, Sabe L, Tiberti C, et al 1997. Cognitive functions in major depression and Parkinson's disease. Arch Neurol, 54:982-6.

Laitinen L. 1969. Desipramine in treatment of Parkinson's disease. Acta Neurol Scand, 45:109-13.

Leentjens AFG, Verhey FR, Lousberg R, et al 2000. The validity of the Hamilton and Montgomery-Asberg depression rating scales as screening and diagnostic tools for depression in Parkinson's disease. Int J Geriatric Psychiatry, 15:644-9.

Leentjens AFG, Verhey FRJ, Luijckx GJ, et al 2000. The validity of the Beck Depression. Inventory as a screening and diagnostic instrument for depression in patients with parkinson's disease. Mov Disord, 6:1221-4.

Leentjens AFG, Vreeling FW, Luijckx GJ, et al 2003. SSRIs in the treatment of depression in Parkinson's disease. Int J Geriatr Psychiatry, 18:552-4.

Lesser RP, Fahn S, Snider SR, et al 1979. Analysis of the clinical problems in parkinsonism and the complications of long term levodopa therapy. Neurology, 29:1253-60.

Levy G, Schupf N, Tang MX, et al 2002. Combined effect of age and severity on the risk of dementia in Parkinson's disease. Ann Neurol, 51:722-9.

Lingjaerde O, Ahlfors UG, Bech Pet al 1987. The UKU side effect rating scale. Acta Psychiatr Scand, 76(Suppl 334):1-99. 
Lombardi WJ, Woolston DJ, Roberts JW, et al 2001. Cognitive deficits in patients with essential tremor. Neurology, 57:785-90.

MacCarthy B, Brown R. 1989. Psychosocial factors in Parkinson's disease. Br J ClinPsychol, 28:41-52.

Mann JJ, Aarons SF, Wilner PJ, et al 1989. A controlled study of the antidepressant efficacy and side effects of (-)-deprenyl. A selective monoamine oxidase inhibitor. Arch Gen Psychiatry, 46:45-50.

Marinus J, Ramaker C, van Hilten JJ, et al 2002. Health related quality of life in Parkinson's disease: a systematic review of disease specific instruments. J Neurol Neurosurg Psychiatry, 72:241-8.

Marsh GG, Markham CH. 1973. Does levodopa alter depression and psychopathology in parkinsonism patients? J Neurol Neurosurg Psychiatry, 36:925-35.

Martin SD, Martin E, Rai SS, et al 2001. Brain blood flow changes in depressed patients treated with interpersonal psychotherapy or venlafaxine hydrochloride: Preliminary finding. Arch Gen Psychiatry, 58:641-8.

Maxmen JS, Ward NG. 1995. Psychotropic drugs: fast facts. New York and London: W.W. Norton and Company.

Mayberg HS, Starkstein SE, Sadzot B, et al 1990. Selective hypometabolism in the inferior frontal lobe in depressed patients with Parkinson's disease. Ann Neurol, 28:57-64.

Mayberg HS, Solomon DH. 1995. Depression in Parkinson's disease: a biochemical and organic viewpoint. Adv Neurol, 65:49-60.

Mayeux R, Stern Y, Rosen J, et al 1981. Depression, intellectual impairment and Parkinson's disease. Neurology, 31:645-50.

Mayeux R. 1984. Behavioral manifestations movement disorders: Parkinson's and Huntington's disease. Neurol Clin, 2:527-40.

Mayeux R, Stern Y, Cote L, et al 1984. Altered serotonin metabolism in depressed patients with Parkinson's disease. Neurology, 34:642-6.

Mayeux R, Stern Y, Williams JBW, et al 1986. Clinical and biomchemical features of depression in Parkinson's disease. Am J Psychiatry, 43:756-9.

Mayeux R. 1992. The mental status in Parkinson's disease. In Koller WC (ed). Handbook of Parkinson's disease. Marcel Decker Inc. p 159-84.

Menza MA, Mark M.H. 1994. Parkinson's disease and depression: the relationship to disability and personality. $J$ Neuropsychiatr Clin Neurosci, 6:165-9.

Meyerowitz BE. 1980. Psychosocial correlates of breast cancer and its treatment. Psychol Bull, 87:108-31.

Mindham RH, Marsden CD, Parkes JD. 1976. Psychiatric symptoms during L-DOPA therapy for Parkinson's disease and their relationship to physical disability. Psychol Med, 6:23-33.

Mindham RH, Ahmed SW, Clough CG. 1982. A controlled study of dementia in Parkinson's disease. J Neurol Neurosurg Psychiatry, 45:969-74.

Moellentine C, Rummans T, Ahlskog EJ, et al 1998. Effectiveness of ECT in patients with parkinsonism. J Neuropsychiatry Clin Neurosci, 10:187-93.

Montastruc JL, Fabre N, Blin O, et al 1995. Does fluoxetine aggravate Parkinson's disease? A pilot prospective study. Mov Disord, 10:355-7.

Montgomery SA, Åsberg M, 1979. A new depression scale designed to be sensitive to change. British Journal of Psychiatry, 134:382-89.

Morrison C, Borod J, Brin M, et al 2000. A program for neuropsychological investigation of Deep Brain Stimulation (PNIDBS) in movement disorder patients: Development, feasibility, and preliminary data. Neuropsychiatry Neuropsychol Behav Neurol, 13:204-19.

Parkinson Study Group. 1993. Effects of tocopherol and deprenyl on the progression of disability in early Parkinson's disease. $N$ Engl J Med, $328: 176-83$.

Paulus W, Trenkwalder C. 1998. Imaging of nonmotor symptoms in Parkinson syndromes. Clinl Neurosci, 5:115-20.

Peto V, Jenkinson C, Fitzpatrick R, et al 1995. The development and validation of a short measure of functioning and well being for individuals with Parkinson's disease. Qual Life Res, 4:241-8.

Poewe W, Luginger E. 1999. Depression in Parkinson's disease: impediments to recognition and treatment options. Neurology, 52:S2-6.
Poewe W, Seppi K. 2001. Treatment options for depression and psychosis in Parkinson's disease. J Neurol, 248:312-21.

Quitkin F, Liebowitz MR, Stewart JW, et al 1984. L-deprenyl in atypical depressives. Arch Gen Psychiatry, 41:777-81.

Rampello L, Chiechio S, Raffaele R, et al 2002. The SSRI, citalopram, improves bradykinesia in patients with Parkinson's disease treated with 1-dopa. Clin Neuropharmacol, 25:21-4.

Raskin S, Borod J, Tweedy J. 1990. Neuropsychological aspects of Parkinson's Disease. Neuropsychol Rev, 1:185-21.

Reichmann H, Brecht MH, Koster J, et al 2003. Pramipexole in routine clinical practice: a prospective observational trial in Parkinson's disease. CNS Drugs, 17:965-73.

Rektorova I, Rector I, Bares M, et al 2003. Pramipexole and pergolide in the treatment of depression in Parkinson's disease: a national multicentre prospective randomized study. Eur J Neurol, 10:399-406.

Richard IH, Kurlan R. 1997. A survey of antidepressant drug use in Parkinson's disease. Parkinson Study Group. Neurology, 49:1168-70.

Robins AH 1976. Depression in patients with parkinsonism. Br J Psychiatry, 128:141-5.

Rubin E, Sackeim HA, Nobler MS, et al 1994. Brain imaging studies of antidepressant treatments. Psychiatr Ann, 24:653-8.

Sagar HJ. 1999. Clinicopathological heterogeneity and non-dopaminergic influences on behavior in Parkinson's disease. Adv Neurol, 80:409-17.

Schrag A, Jahanshahi M, Quinn NP. 2001. What contributes to depression in Parkinson's disease? Psychol Med, 31:65-73.

Scott B, Borgman H, Engler B, et al 2000. Gender differences in Parkinson's disease symptom profile. Acta Neurol Scand, 102:37-43.

Schrag A, Jahanshahi M, Quinn NP. 2001. What contributes to depression in Parkinson's disease? Psychol Med, 31:65-73.

Singer E. 1976. Sociopsychological factors influencing response to levodopa therapy for Parkinson's disease. Arch Phys Med Rehabil, 57:328-34.

Slaughter JR, Slaughter KA, Nichols D, et al 2001. Prevalence, clinical manifestations, etiology, and treatment of depression in Parkinson's disease. J Neuropsychiatry Clin Neurosci, 13:187-96.

Starkstein SE, Preziosi TJ, Bolduc PL, et al 1990. Depression in Parkinson's disease. J Nerv Ment Dis, 178:37-41.

Stern Y, Marder K, Tang MX, et al 1993. Antecedent clinical features associated with dementia in Parkinson's disease. Neurology, 43:1690-2.

Steur EN. 1993. Increase of Parkinson disability after fluoxetine medication. Neurology, 43:211-13.

Strome EM, Zis, AP, Doudet DJ. 2007. Electroconvulsive shock enhances striatal dopamine $\mathrm{D}(1)$ and $\mathrm{D}(3)$ receptor binding and improves motor performance in 6-OHDA-lesioned rats. $J$ Psychiatry Neurosci, $32: 193-202$.

Srinivasan J, Schmidt WJ. 2003. Potentiation of parkinsonian symptoms by depletion of locus coeruleus noradrenaline in 6-hydroxydopamineinduced partial degeneration of substantia nigra in rats. Eur J Neurosci, 17:2586-92.

Srinivasan J, Schmidt WJ. 2004. Treatment with alpha2-adrenoceptor antagonist, 2-methoxyidazoxan, protects 6-hydroxydopamine-induced parkinsonian symptoms in rats: neurochemical and behavioral evidence. Beh Brain Res, 154:353-63.

Strang RR. 1965. Imipramine in treatment of Parkinsonism: a double-blind placebo study. BMJ, 2:33-4.

Sunderland T, Muller EA, Cohen RM, et al 1985. Tyramine pressor sensitivity changes during deprenyl treatment. Psychopharmacol, 86:432-37.

Tandberg E, Larsen JP, Aarsland D, et al 1997. Risk factors for depression in Parkinson's disease. Arch Neurol, 54:625-30.

Tesei S, Antonini A, Canesi M, et al 2000. Tolerability of paroxetine in Parkinson's disease: a prospective study. Mov Disord, 15:986-9.

Trend P, Kaye J, Gage H, et al 2002. Short-term effectiveness of intensive multidisciplinary rehabilitation for people with Parkinson's disease and their carers. Clin Rehabil, 16:717-25.

Uekermann J, Daum I, Peters S, et al 2003. Depressed mood and executive dysfunction in early Parkinson's disease. Acta Neurol Scand, 107:341-8. 
Warburton JW. 1967. Depressive symptoms in Parkinson patients referred for thalamotomy. J Neurol Neurosurg Psychiatry, 30:368-70.

Weintraub D, Morales KH, Moberg PJ, et al 2005: Antidepressant studies in Parkinson's disease: a review and meta-analysis. Mov Disord, 20:1161-9.

Wermuth L, Sorensen PS, Timm S, et al 1998. Depression in idiopathic Parkinson's disease treated with citalopram. Nord J Psychiatry, 52:163-9.
Yesavage JA, Brink TL, Rose TL, et al 1982. Development and validation of a geriatric depression rating scale: a preliminary report. $J$ Psych Res, 17:37-49.

Zesiewicz TA, Gold M, Chari G, et al 1999. Current issues in depression in Parkinson's disease. Am J Geriatr Psychiatry, 7:110-18.

Zgaljardic D, Borod J, Foldi N, et al 2003. A review of the cognitive and behavioral sequelae of Parkinson's disease: Relationship to frontostriatal circuitry. Cogn Behav Neurol, 16:193-210. 
